\title{
Les problèmes d'eutrophisation littorale et leur modélisation
}

\section{Eutrophication in the coastal zone and its modelling}

\author{
Alain Menesguen \\ IFREMER Centre de Brest, Direction de l'Environnement Littoral, B.P. 70, 29280 Plouzané - \\ France
}

Résumé. - Après avoir d'abord frappé les lacs, puis les rivières, l'eutrophisation engendrée par l'augmentation des apports terrigènes de sels nutritifs azotés et phosphorés a atteint depuis vingt ans de nombreux estuaires dans le monde, et même de vastes zones côtières peu profondes depuis une dizaine d'années. L'étude in situ et la modélisation de deux cas d'eutrophisation rencontrés sur les côtes bretonnes (France), permet de comprendre pourquoi l'enrichissement excessif d'une zone littorale peut conduire soit à une prolifération de macroalgues (chlorophycées), soit à une pullulation phytoplanctonique. Dans les deux cas est mis en évidence le rôle crucial du temps de résidence des masses d'eau dans la zone littorale enrichie. En milieu littoral, la difficulté d'établir aussi bien la valeur de ce temps de résidence que la nature du facteur limitant (azote, phosphore ou lumière) rend peu fiable l'utilisation des abaques statistiques pourtant si efficaces dans la gestion de l'eutrophisation des lacs; l'intérêt des modèles numériques dynamiques en zone marine est donc examiné et discuté.

Mots-clés. - Eutrophisation côtière, élément limitant, temps de résidence, modèles hydrodynamiques, modèles écologiques.

Abstract. - Following lakes and rivers, a lot of estuaries (during the last twenty years) and shallow coastal zones (during the last decade) have been severely eutrophicated because of the too much increasing loads of nitrogen and phosphorus. In situ study and numerical modelling of two French case studies bring some explanation to the fact that, in some sites, enrichment turns into green macroalgal proliferation whereas, in others, mass blooms of phytoplankton are induced. Whatever the case may be, the prominent role of the residence time of water in the enriched zone has been proven. In open coastal ecosystems, the difficulty in determining this residence time as well as the limiting factor (nitrogen, or phosphorus, or light) lowers considerably the reliability of the classical 
statistical diagrams which have been used successfully in management of eutrophicated lakes. The numerical dynamic modelling provides a promising alternative, which is discussed.

Key-words. - Coastal eutrophication, Limiting factor, Residence time, Hydrodynamical models, Ecological models.

\section{INTRODUCTION}

L'utilisation intense des fertilisants artificiels en agriculture (engrais minéraux, lisiers) ainsi que l'accroissement des rejets urbains ont augmenté notablement depuis trente ans les apports terrigènes d'éléments nutritifs au milieu aquatique. En de nombreux points du globe, cet enrichissement forcé en nitrate (issu principalement du lessivage des terres agricoles), en ammonium et phosphate (très abondants dans les rejets urbains), a causé un déséquilibre du milieu aquatique, appelé eutrophisation, qui se manifeste par une production algale pléthorique suivie souvent de chutes d'oxygène dissous préjudiciables à la faune. Les lacs, à renouvellement très lent, ont été historiquement les premiers touchés (Grands Lacs américains, lac du Bourget,...) et ont suscité de nombreuses études dans le but de proposer un scénario optimal de restauration de la qualité du milieu. Un effort important de modélisation écologique a en particulier été mené aux États-Unis (Thomann et al., 1975; Park, 1978; di Toro et Connolly, 1980) et au Danemark (Jorgensen, 1980). Puis les grands cours d'eau, apparemment moins vulnérables du fait de l'écoulement de l'eau, ont été largement touchés, suscitant là-encore un effort important de compréhension des mécanismes et de modélisation; on peut citer en France le modèle POLUPA d'eutrophisation de la Loire (Oudin, 1990), ceux de la Moselle et du Doubs (Gosse et al., 1990). De toutes ces études, le résultat le plus marquant, car vérifié sur la majeure partie des écosystèmes limniques eutrophisés dans le monde, est la mise en évidence du phosphore comme étant l'élément limitant de la production végétale dans les eaux douces (Schindler et al., 1978). Plus récemment, le phénomène d'eutrophisation a atteint les grands estuaires (Loire, Potomac, Patuxent,...) et les baies côtières (Chesapeake Bay, San Francisco Bay, New-York Bight,...), voire même de vastes zones marines peu profondes, influencées par les apports de grands fleuves (partie orientale de la Mer du Nord, Baltique, nord de l'Adriatique,...). Comme en eau douce, l'une des conséquences les plus fâcheuses de l'accumulation près du fond de fortes quantites de phytoplancton privé de lumière est l'épuisement rapide de l'oxygène dissous dans l'eau, par respiration et décomposition du phytoplancton. Le phénomène d'anoxie mortelle peut s'étendre alors sur de vastes zones 
marines: Baltique (Dethlefsen et Von Westernhagen, 1983), New-York Bight (Swanson et Sindermann, 1979), Golfe de Trieste (Stachowitsch, 1984). La complexité de l'environnement marin a cependant freiné l'élaboration de modèles écologiques d'eutrophisation côtière, d'où le petit nombre de ces travaux publiés dans les années 80 : Fransz et Verhagen (1985), Mommaerts et al., (1984) pour la Mer du Nord, Malmgren-Hansen et al. (1984) dans le Limfjord au Danemark, Humphries et al. (1984) pour le Peel Inlet (Australie)... Les côtes françaises, généralement peu touchées pour l'instant par les phénomènes d'eutrophisation massive, montrent cependant quelques cas de sites localement très perturbés, certains par des proliférations de macroalgues vertes (ulves), d'autres par des pullulations phytoplanctoniques. L'étude par modélisation écologique de ces deux types d'eutrophisation permet de mieux comprendre la genèse de ces nuisances et de formuler des recommandations par tenter de les diminuer.

\section{L'EUTROPHISATION COTIERE A MACROALGUES}

\subsection{Présentation succincte du phénomène}

Phénomène connu de tout temps et sans doute caractéristique de l'écologie "naturelle" de ces végétaux marins, la prolifération printanière et estivale de certaines macroalgues vertes (ulves, entéromorphes, cladophores) a cependant pris une ampleur alarmante depuis une vingtaine d'années sur certains sites particuliers. Les zones les plus favorables à cette prolifération macroalgale sont bien sûr les lagunes semi-fermées peu profondes et sous éclairement solaire intense: c'est ainsi que la lagune de Venise a présenté jusqu'à 550000 tonnes d'ulves (poids frais) en juin 1987 (Striso et al., 1989) et que dans le Lac de Tunis se développait avant les récents aménagements hydrauliques, une biomasse estivale fluctuant entre 13000 et 44000 tonnes pour la période 1975-1978 (Kelly et Naguib, 1984). Plus étonnantes sont les proliférations d'algues vertes parfois très spectaculaires que l'on peut rencontrer sur certaines plages apparemment largement ouvertes sur la mer et soumises à des marées significatives. Des "marées vertes" à cladophores réapparaissent ainsi tous les étés depuis 1976 sur les plages du Kattegat en Suède (Baden et al., 1990) alors que ce sont les ulves qui forment de gigantesques échouages sur les plages des côtes bretonnes (Piriou et al., 1989), ainsi que le révèlent les tonnages collectés annuellement par les communes littorales (fig. 1). Le cas des "marées vertes" bretonnes est d'autant plus étrange que la classique corrélation observée entre ces proliférations et l'enrichissement local des eaux par des apports terrigènes de sels azotés (Letts et Richards, 1991 ; Sawyer, 1965, Steffensen, 1976), ne semble plus jouer, au vu de la répartition géographique 
comparée des apports annuels de nitrates et des biomasses d'algues échouées (fig. $2 a$ et $2 b$, Piriou et al., 1989). Une étude poussée d'un site, la Baie de Saint-Brieuc, a permis l'élaboration d'un modèle numérique du phénomène, dont l'examen ci-après montrera les applications pratiques.

\subsection{Le modèle hydrodynamique}

Située sur les côtes de la Manche (fig. 3), la Baie de Saint-Brieuc est soumise à un fort régime de marée semi-diurne, qui génère des courants calculables par un modèle hydrodynamique bidimensionnel horizontal (Le-

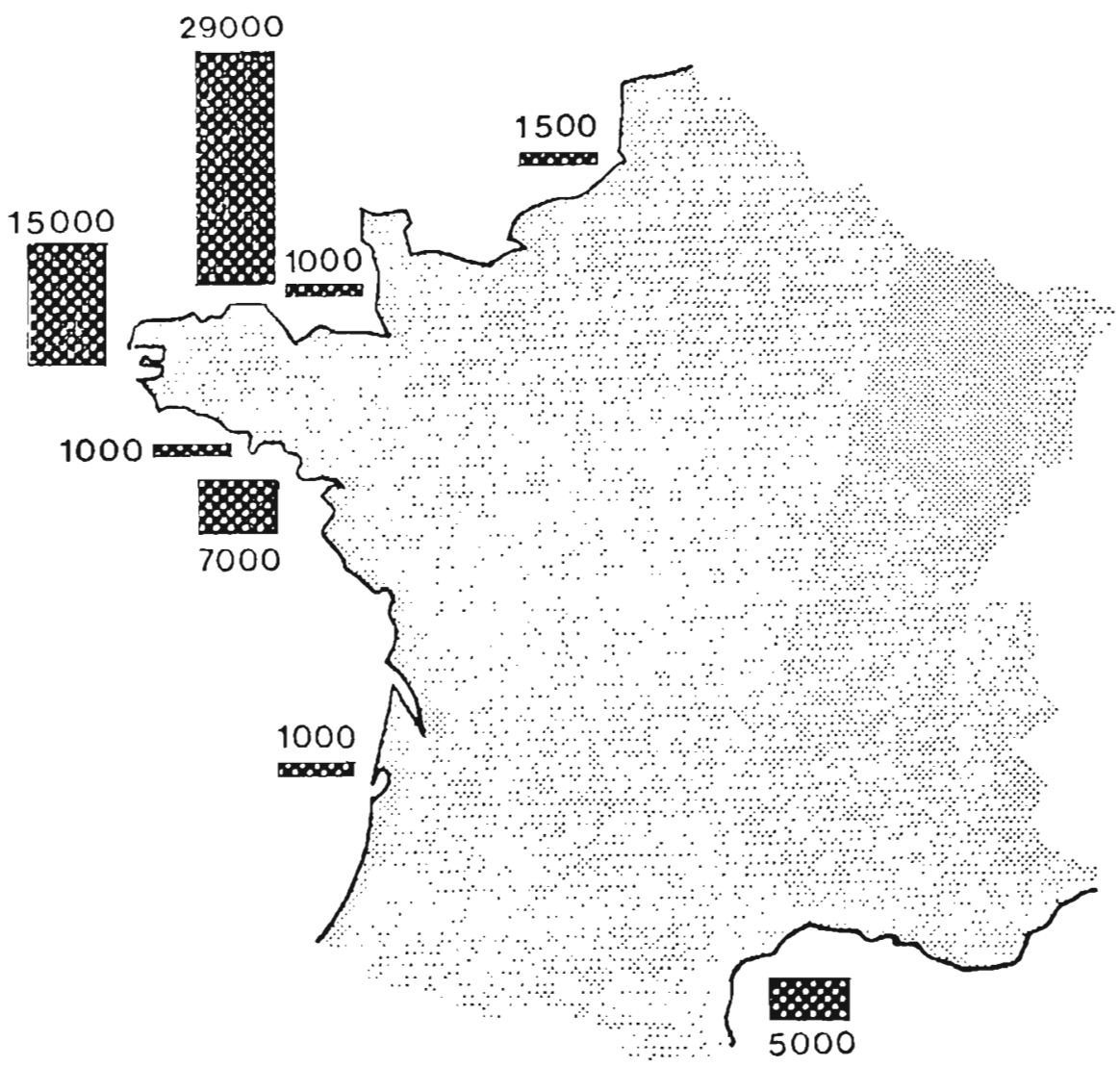

Fig. 1. - Volume $\left(\mathrm{m}^{3}\right)$ d'algues vertes collectés en 1987 par les communes littorales.

Fig. 1. - Volumes $\left(\mathrm{m}^{3}\right)$ of green macroalgae collected during 1987 by municipalities. 

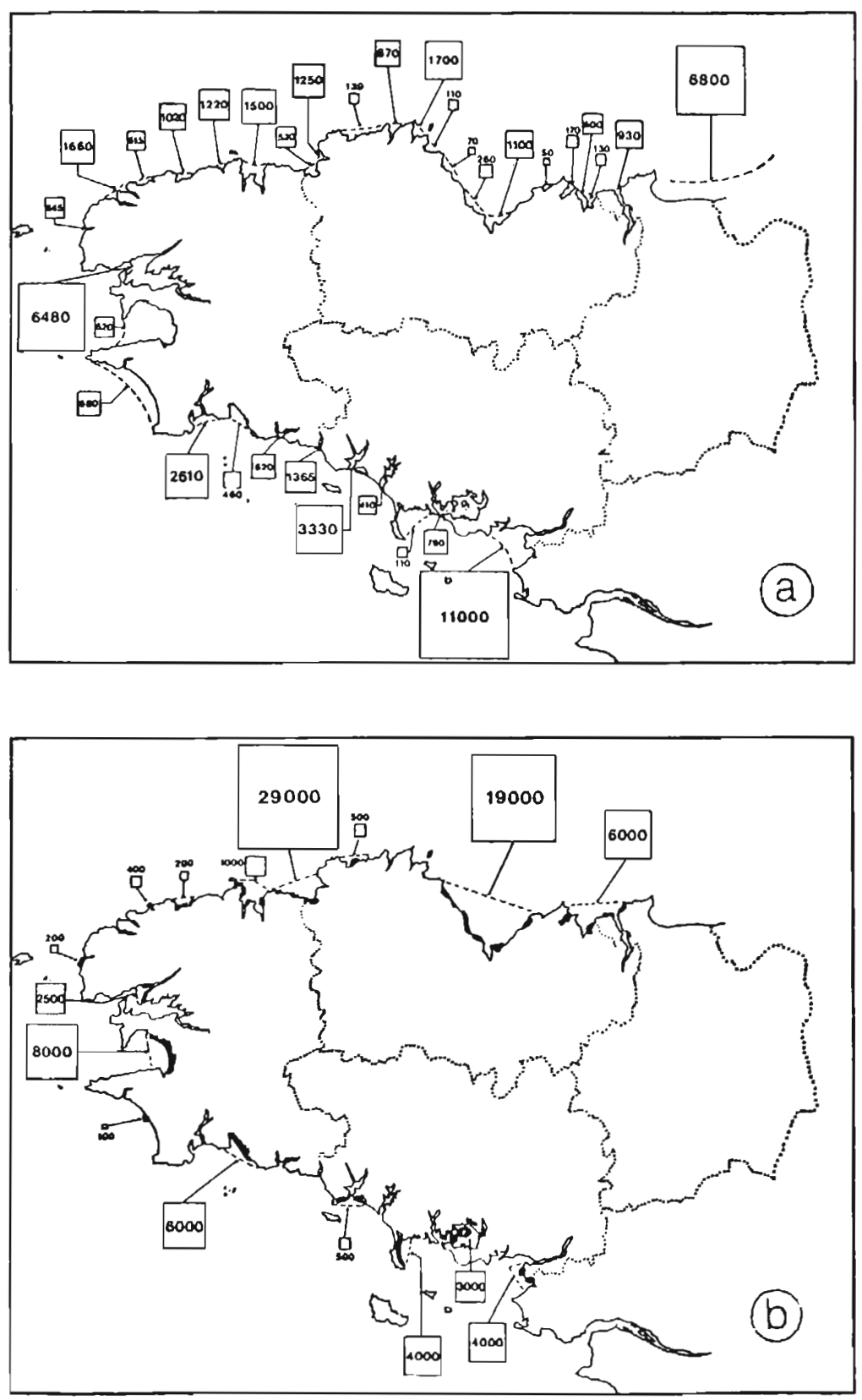

Fig. 2a. - Apports terrigènes de $\mathrm{N}-\mathrm{NO}_{3}$ (tonnes/an) en 1984 sur les côtes bretonnes

Fig. 2a. - N-NO $\mathrm{N}_{3}$ loadings (tons/year) during 1984 along the coast of Brittany.

Fig. 2b. - Volumes $\left(\mathrm{m}^{3}\right)$ d'algues vertes collectées en 1984 sur les côtes bretonnes.

Fig. 2b. - Volumes $\left(\mathrm{m}^{3}\right)$ of green macroalgae collected during 1984 along the coast of Brittany. 


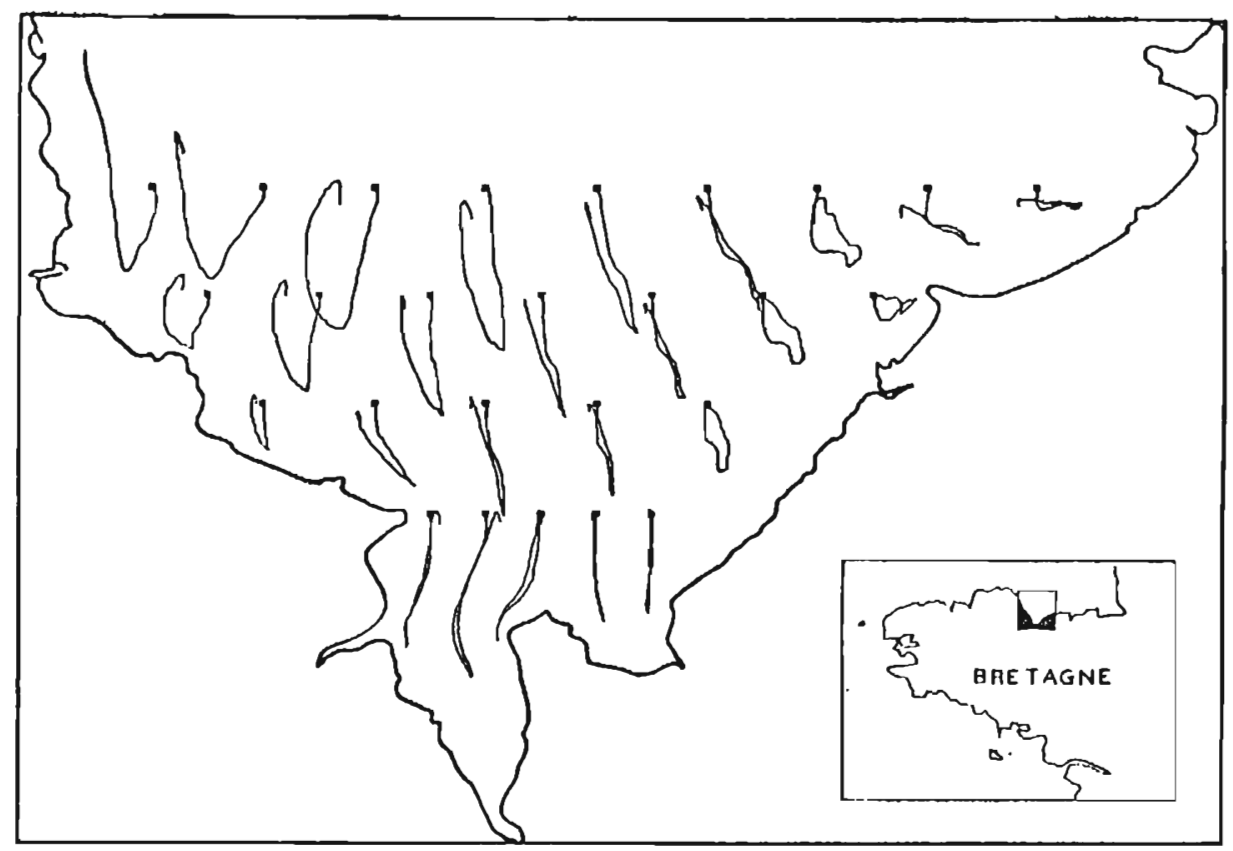

Fig. 3. - Trajectoires de particules entre deux basses mers consécutives en Baie de Saint-Brieuc.

Fig. 3. - Calculated drifts between two consecutive low tides in the Bay of Saint-Brieuc.

hay, 1989). Ceux-ci permettent de construire les trajectoires lagrangiennes que suivraient des flotteurs largués aux nœuds du maillage (Ménesguen et Salomon, 1988). II apparaît alors des zones, ici au nord-ouest (fig. 3), où ces flotteurs subiront une dérive résiduelle importante de marée en marée, alors qu'au sud de la baie, les flotteurs reviendront exactement à leur point de départ au bout d'une marée $(12 \mathrm{~h} 24 \mathrm{~mm})$. Ainsi que l'ont montré des photographies aériennes, la plus grande partie des ulves en suspension dans l'eau se trouve justement confinée dans la zone de balan- cement des marées où la résiduelle de marée s'annule quasiment (fig. 4). La modélisation numérique de la circulation résiduelle de marée (Salomon et al., 1988) apporte donc l'explication du paradoxe apparent des proliférations végétales sur estran macrotidal et largement ouvert vers la haute mer: il s'agit dans ce cas d'un confinement dynamique local généré par l'interaction de l'onde de marée et de la topographie du fond. Le fait que le confinement intervienne dans une zone très peu profonde et sableuse assure un éclairement très intense de la tranche d'eau, et permet 


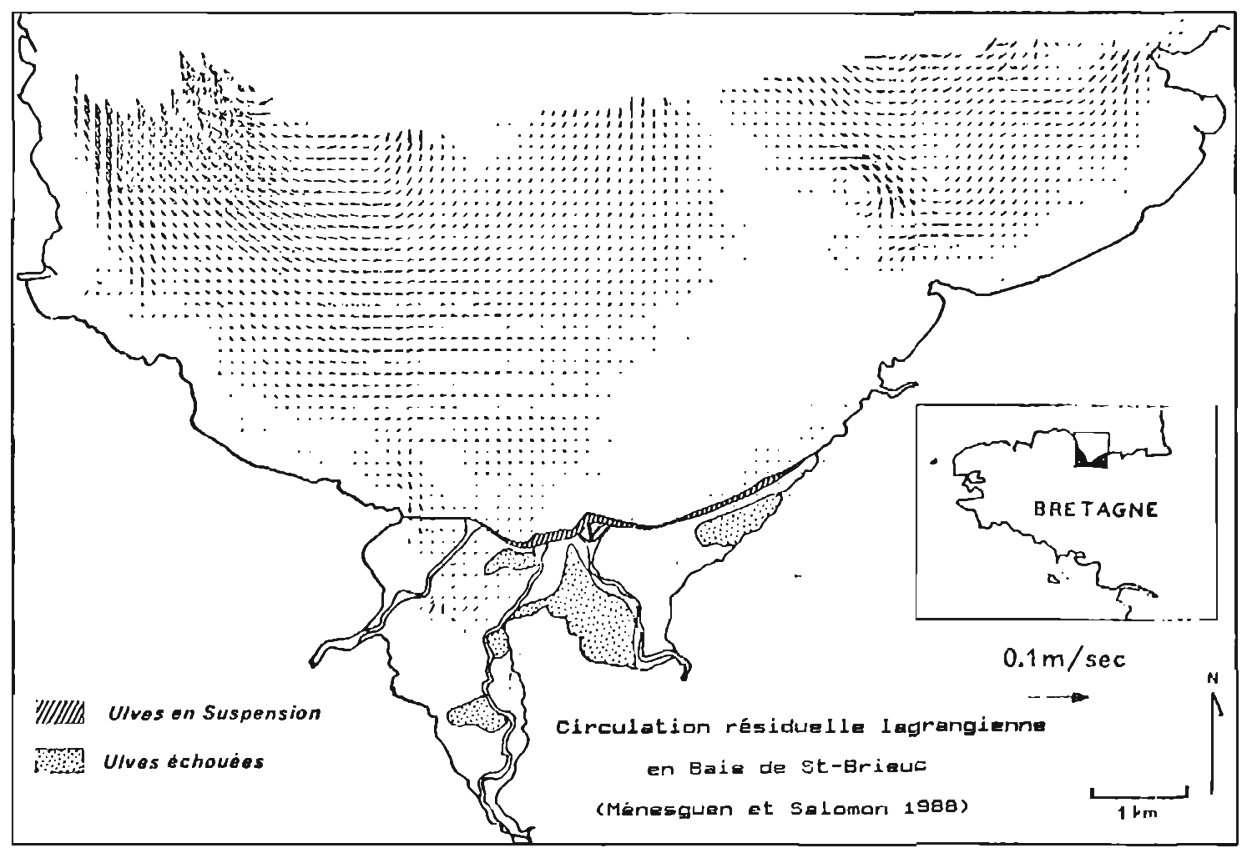

Fig. 4. - Champ de courants résiduels et lieux de prolifération d'ulves en Baie de Saint-Brieuc. Fig. 4. - Field of residual currents and localization of ulva accumulations in the Bay of Saint-Brieuc.

un bon brassage de la suspension d'algues, deux conditions indispensables à la photosynthèse des ulves. Pour les besoins ultérieurs de la modélisation écologique, la zone utile est découpée en grands compartiments supposés homogènes (fig. 5), entre lesquels l'advection est calculée d'après le champ de courants résiduels précédent et la dispersion estimée, d'après l'excursion de marée.

\subsection{Le modèle biologique}

II doit contenir les seules variables d'état nécessaires et suffisantes pour décrire les processus majeurs conduisant à la prolifération. Dans le cas des «marées vertes» sur estran sableux macrotidal, l'absence d'espèce macroalgale compétitrice (monoculture d'ulves), des prédateurs herbivores habituels (gastéropodes rampants) ainsi que de stockage à long terme d'éléments nutritifs par le sédiment autorise à ne retenir que les cinq variables d'état suivantes : azote inorganique total dissous dans l'eau de mer, phosphore inorganique total dissous dans l'eau de mer, azote total contenu dans les ulves, phosphore total contenu dans les ulves, biomasse totale des ulves. 


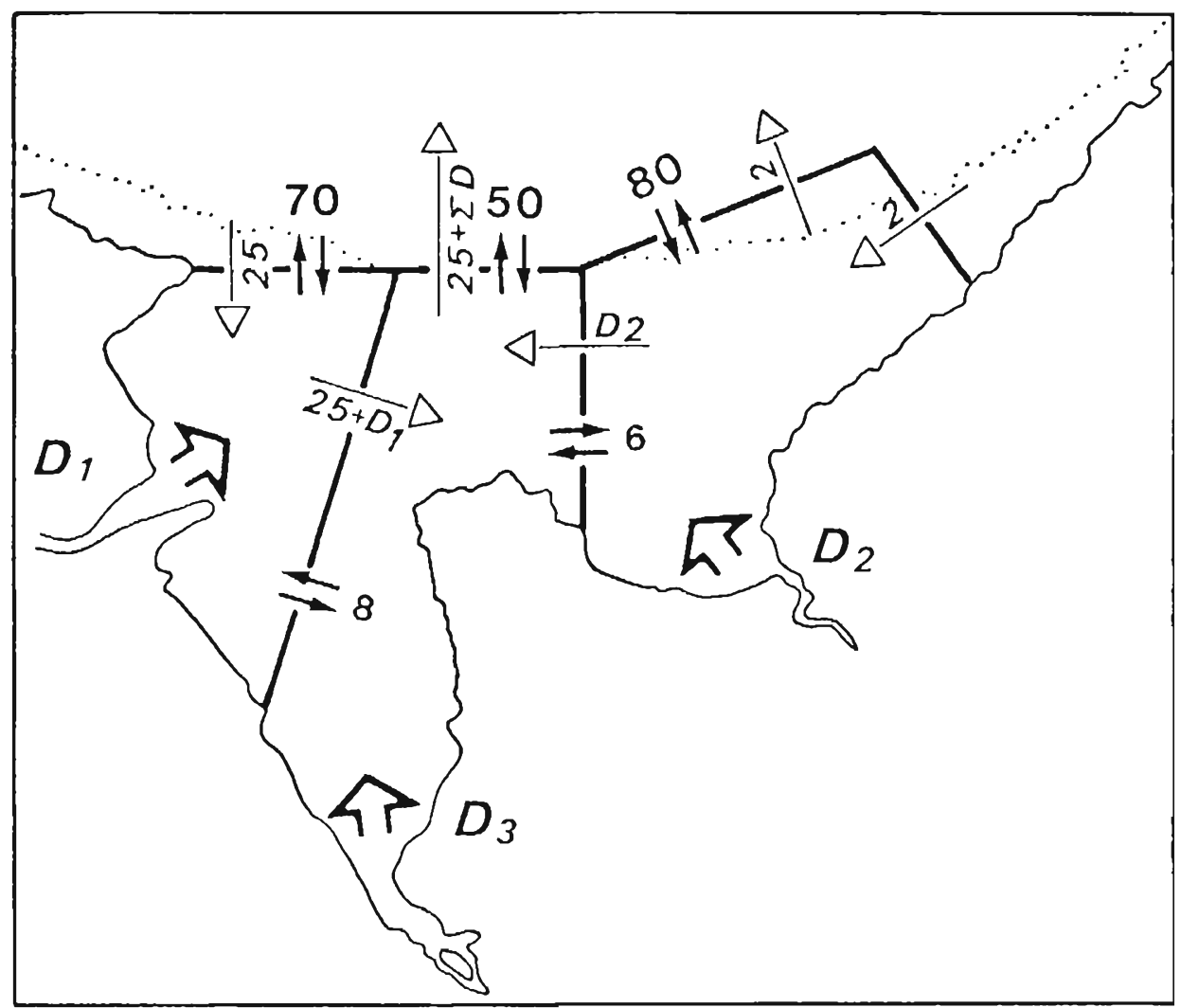

Fig. 5. - Découpage du sud de la Baie de Saint-Brieuc en compartiments géographiques et flux en $\mathrm{m}^{3} \cdot \mathrm{s}^{-1}$.

Fig. 5. - Box model of the southern part of the Bay of Saint-Brieuc with hydraulic fluxes in $\mathrm{m}^{3} \cdot \mathrm{s}^{-1}$.

La simulation explicite du contenu des algues en $\mathrm{N}$ et $\mathrm{P}$ correspond à la volonté de prendre en compte la grande capacité de stockage des sels nutritifs par les algues (Fujita, 1985) et de pouvoir comparer la simulation du carencement en $\mathrm{N}$ et/ou $\mathrm{P}$ aux mesures effectivement réalisées sur le terrain (Dion, 1988). II s'agit donc d'un modèle de croissance dit "à quotas" (Droop, 1970), influencé de façon classique par les variables forçantes lumière et température (fig. 6). Le détail des équations et de leurs paramètres est fourni dans Ménesguen (1992). 


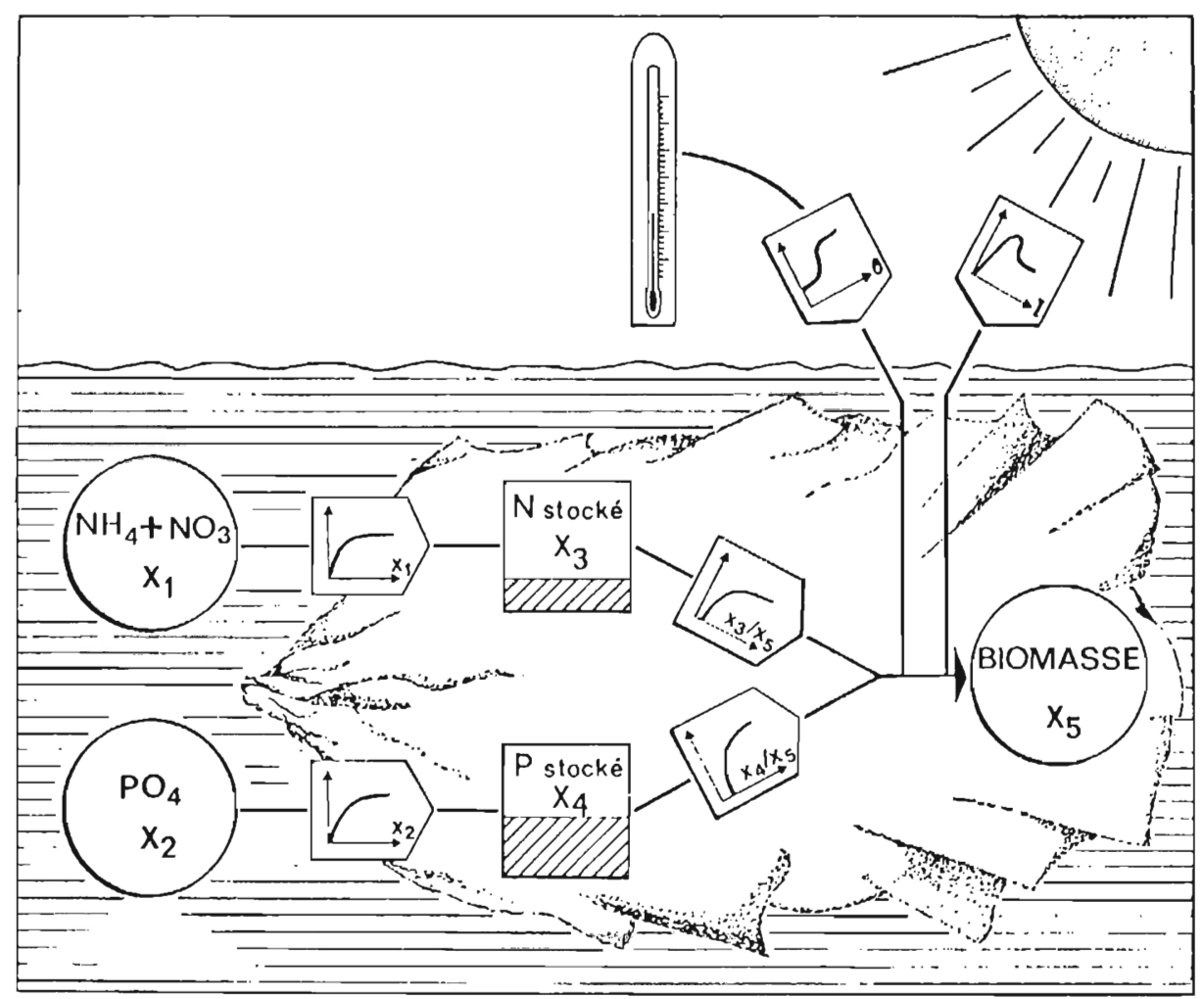

Fig. 6. - Schéma fonctionnel du modèle biologique de prolifération d'ulves.

Fig. 6. - Flow diagram of the biological model for ulva proliferation.

\subsection{Résultats}

Le modèle écologique global, résultant du couplage entre le modèle hydrodynamique à compartiments et le modèle biologique précédent, peut d'abord être utilisé pour reproduire le cycle annuel moyen actuellement observé. Sous forçage thermique et lumineux strictement périodique (période annuelle), la biomasse totale simulée pour le sud de la Baie de Saint-Brieuc suit une évolution saisonnière ellemême de période annuelle, caractérisée par la brusque prolifération printanière suivie d'une décroissance estivale lente (fig. 7). Ceci reproduit bien la forme des courbes d'évolution de la biomasse effectivement mesurées sur le terrain (fig. 8). Ces dernières montrent toutefois une hauteur de pic en juillet très variable selon les années. Piriou et Ménesguen (1990) ont pu corréler la hauteur du pic es- 


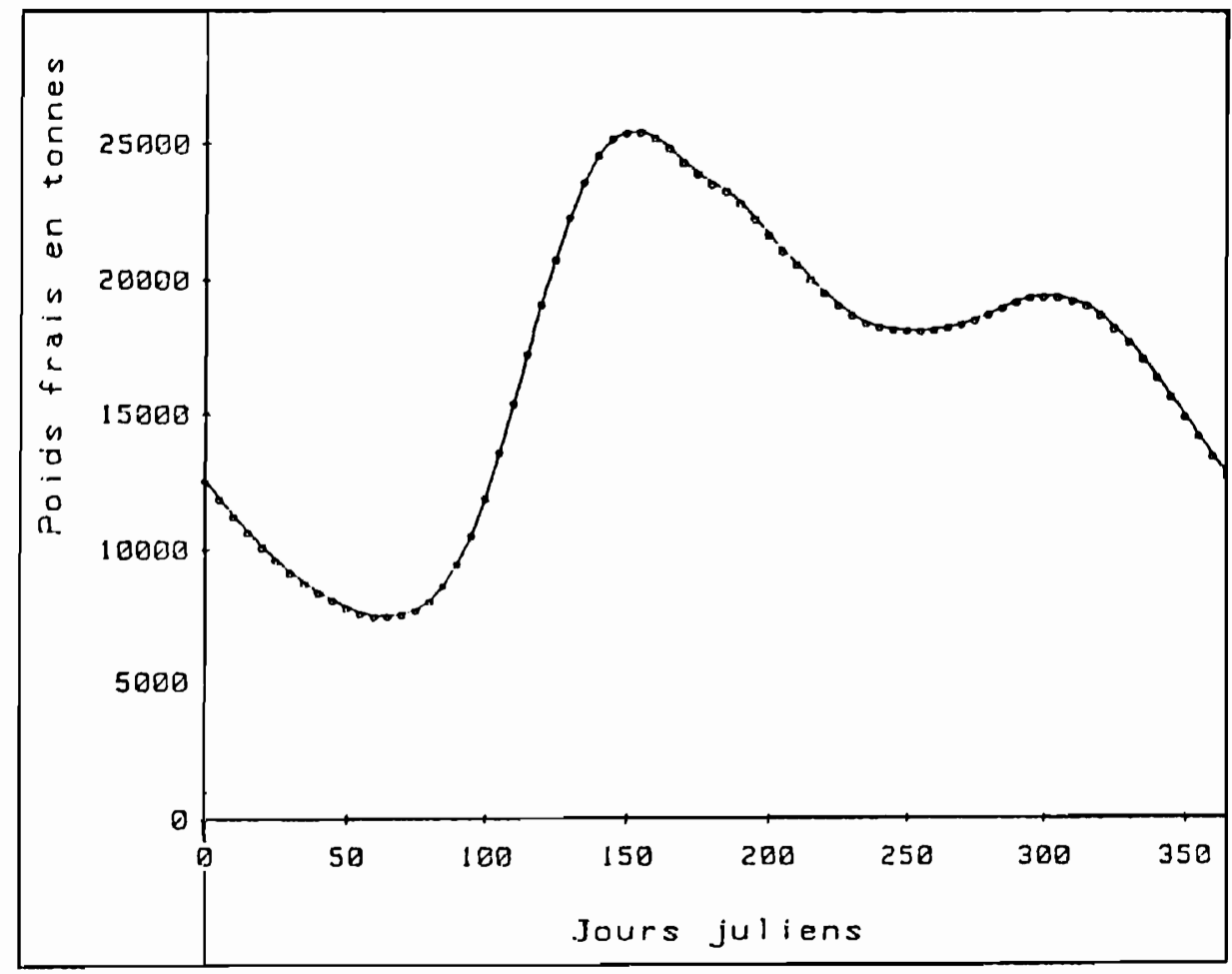

Fig. 7. - Evolution saisonnière simulée de la biomasse totale d'ulves dans le sud de la Baie de Saint-Brieuc (2400 ha) pour une année météorologique moyenne.

Fig. 7. - Simulated seasonal evolution of the total ulva biomass in the southern part of the Bay of Saint-Brieuc during a mean meteorological year.

tival de biomasse d'ulves avec le flux total d'azote amené par ces rivières durant le seul mois de juin, c'est-àdire pendant la phase de croissance maximale. Plus généralement, il est intéressant de tester la réponse du modèle de prolifération d'ulves à des scénarios très différents d'apports terrigènes. L'année 1982 par exemple est particulièrement pluvieuse en été et automne et la concentration d'azote inorganique dans les rivières y fluctue entre 250 et 450 micromoles $\mathrm{N} /$ litre. L'année 1988 est par contre une année très sèche à partir du mois de mai, mais les concentrations en azote inorganique ont doublé (variant entre 450 et 850 micromoles $\mathrm{N} /$ /itre) du fait de l'usage accru de fertilisants. Les concentrations de phosphore inorganique sont restées dans la même plage de variation. On constate que le mo- 


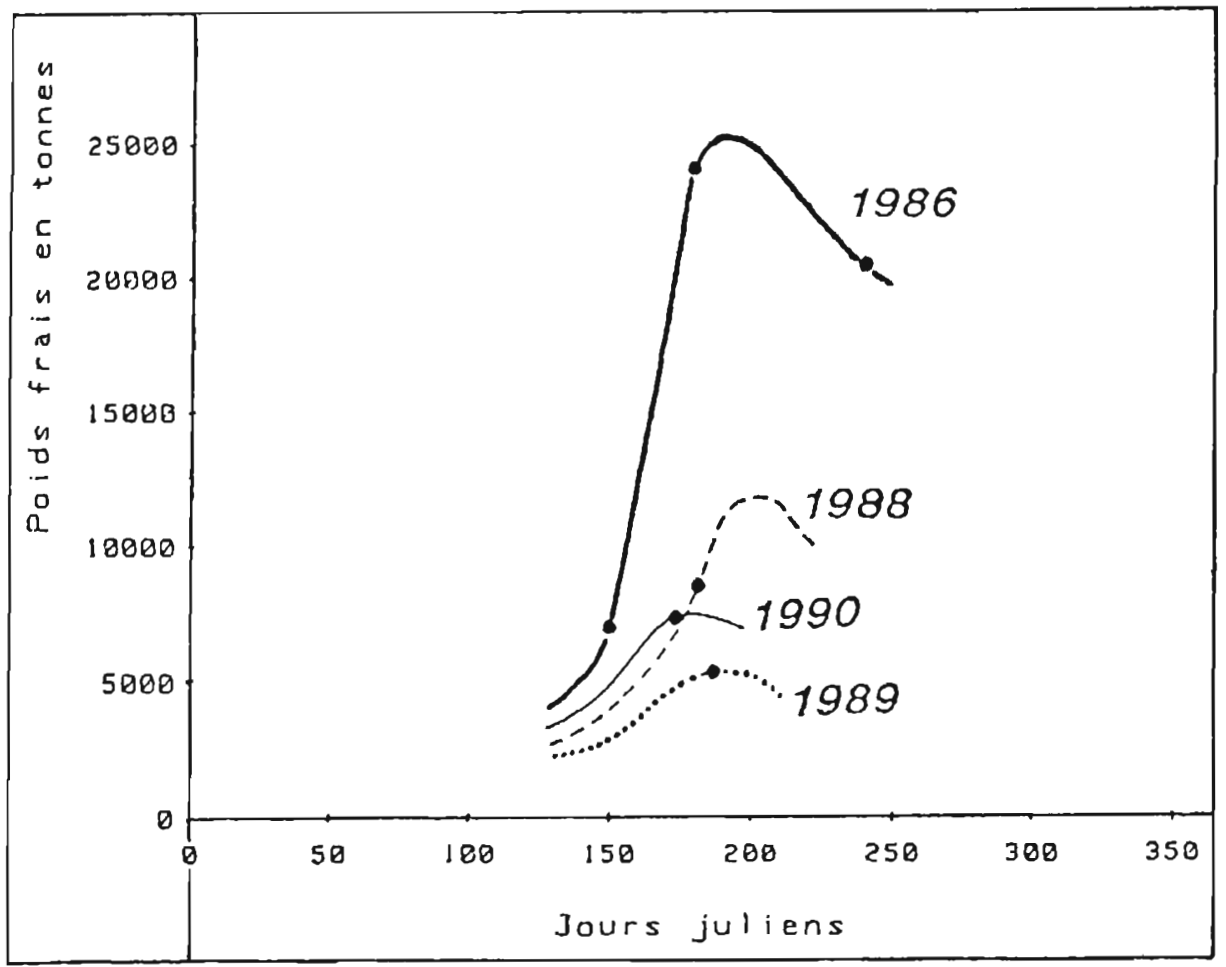

Fig. 8. - Evolution printanière mesurée de la biomasse totale d'ulves dans le sud de la Baie de Saint-Brieuc (2400 ha).

Fig. 8. - Observed spring evolution of the total ulva biomass in the southern part of the Bay of Saint-Brieuc (2400 ha).

dèle réagit fortement à ces deux scénarios annuels de flux terrigènes, et produit un pic estival de biomasse sensiblement double en 1988 qu'en 1982 (fig. 9). L'examen des flux de nutrients pendant la seule période printanière fait effectivement ressortir l'apport double d'azote en 1988 par rapport à 1982, bien que la deuxième moitié de l'année ait été sèche. Le rôle clé de l'azote dans le contrôle de ces proliférations algales, déjà clairement montré par Sfriso et al., (1989) dans la lagune de Venise, est ainsi retrouvé sur les côtes bretonnes. Le modèle numérique peut d'ailleurs servir à préciser de façon très concrète le rôle respectif de l'azote et du phosphore dans le contrôle de la prolifération des ulves: sur la surface de réponse du pic estival de biomasse à des diminutions variées des apports 


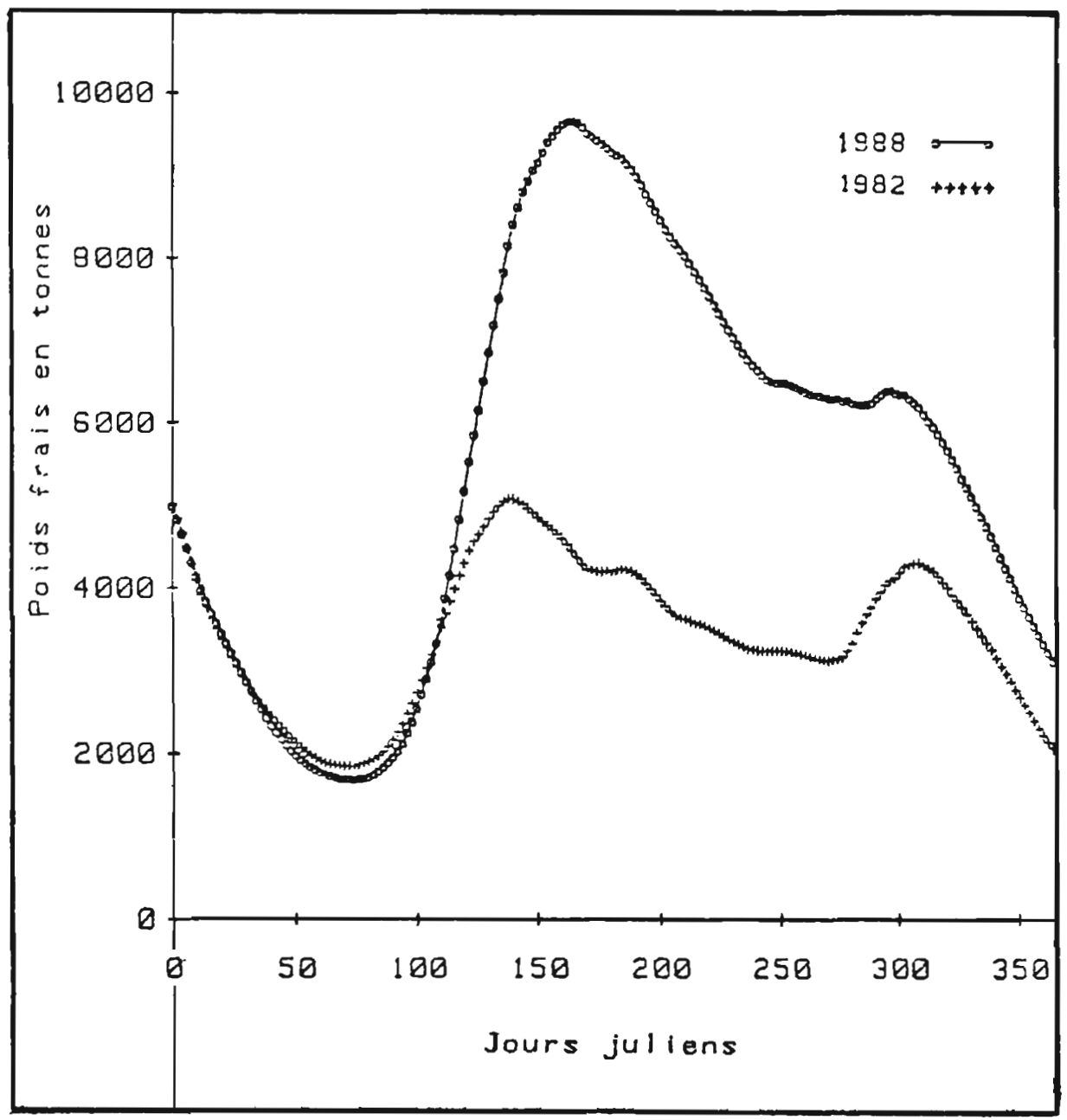

Fig. 9. - Evolution saisonnière simulée de la biomasse totale d'ulves pour les années 1982 et 1988 dans le sud de la Baie de Saint-Brieuc (2400 ha).

Fig. 9. - Simulated seasonal evolution of total ulva biomass during 1982 et 1988 in the southern part of the bay of Saint-Brieuc (2400 ha).

terrigènes d'azote et/ou de phosphore (fig. 10), on constate bien l'effet majeur sur la réduction de la prolifération obtenu en diminuant les seuls apports d'azote. Le modèle peut donc fournir des arguments quantitatifs pour orienter la politique de lutte contre cette forme d'eutrophisation littorale. 


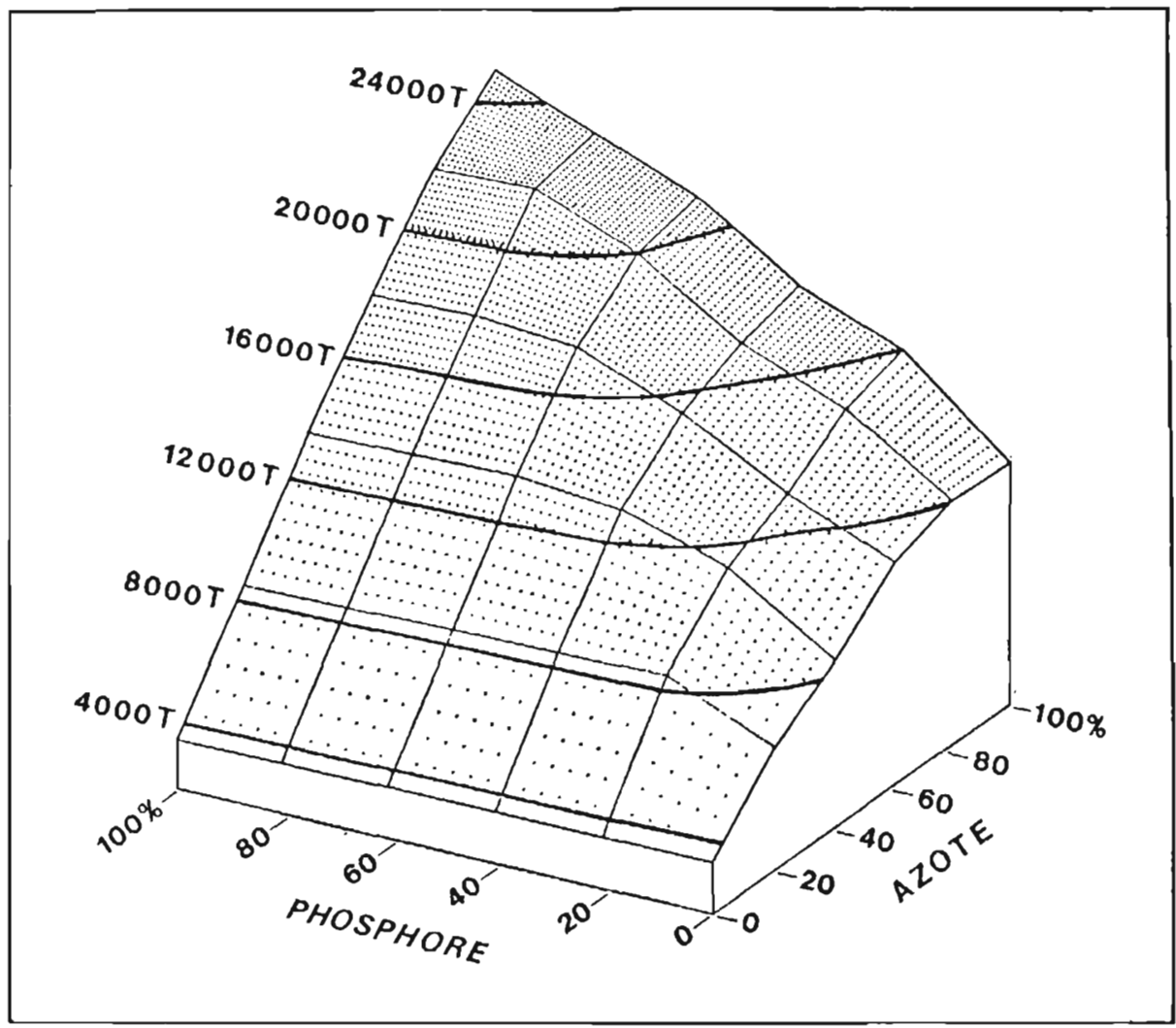

Fig. 10. - Réponse du pic estival de biomasse d'ulves à des réductions variées des apports de $N$ etlou $P$.

Fig. 10. - Response surface of the summer pike of total ulva biomass to various reduced $\mathrm{N}$ and/or $P$ river loadings.

\section{L'EUTROPHISATION COTIĖRE À PHYTOPLANCTON}

\subsection{Les caractéristiques de ce phénomène}

Le scénario général est toujours identique, même si les espèces phytoplanctoniques impliquées peuvent être différentes d'un site à l'autre ou d'une année sur l'autre. Alors qu'en mer tempérée, il y a en général une seule floraison majeure du phytoplancton, au printemps, qui épuise le stock disponible de sels nutritifs, les zones côtières soumises à d'importants apports issus d'une rivière ou d'un fleuve peuvent soutenir plusieurs floraisons intenses durant le prin- 
temps et l'été grâce à leur approvisionnement continu en nutrients. L'extrême abondance du phytoplancton (des millions, voire des dizaines de millions de cellules par litre) obscurcit alors l'eau, empêchant toute photosynthèse sur une épaisseur de plusieurs mètres au dessus du fond et provoquant une intense respiration puis une dégradation aérobie du phytoplancton. Si le réapprovisionnement en oxygène de l'air n'est pas assuré par un brassage suffisant de la tranche d'eau, par exemple parce qu'une stratification haline ou thermique s'est établie, l'anoxie peut inter- venir en quelques heures et asphyxier la faune benthique. Si certains estuaires connaissent cette situation de façon endémique, comme la zone médiane de la Chesapeake Bay (fig. 11) décrite par Officier et al. (1984), les anoxies mortelles en zone marine sont plutôt des événements catastrophiques sporadiques, liés à une conjoncture fortuite de plusieurs facteurs propices, ainsi qu'il a été prouvé (Merceron, 1987) pour l'anoxie mortelle survenue en juillet 1982 en Baie de Vilaine (côte Atlantique française). La modélisation prévisionnelle de ce type d'événements s'avère donc très

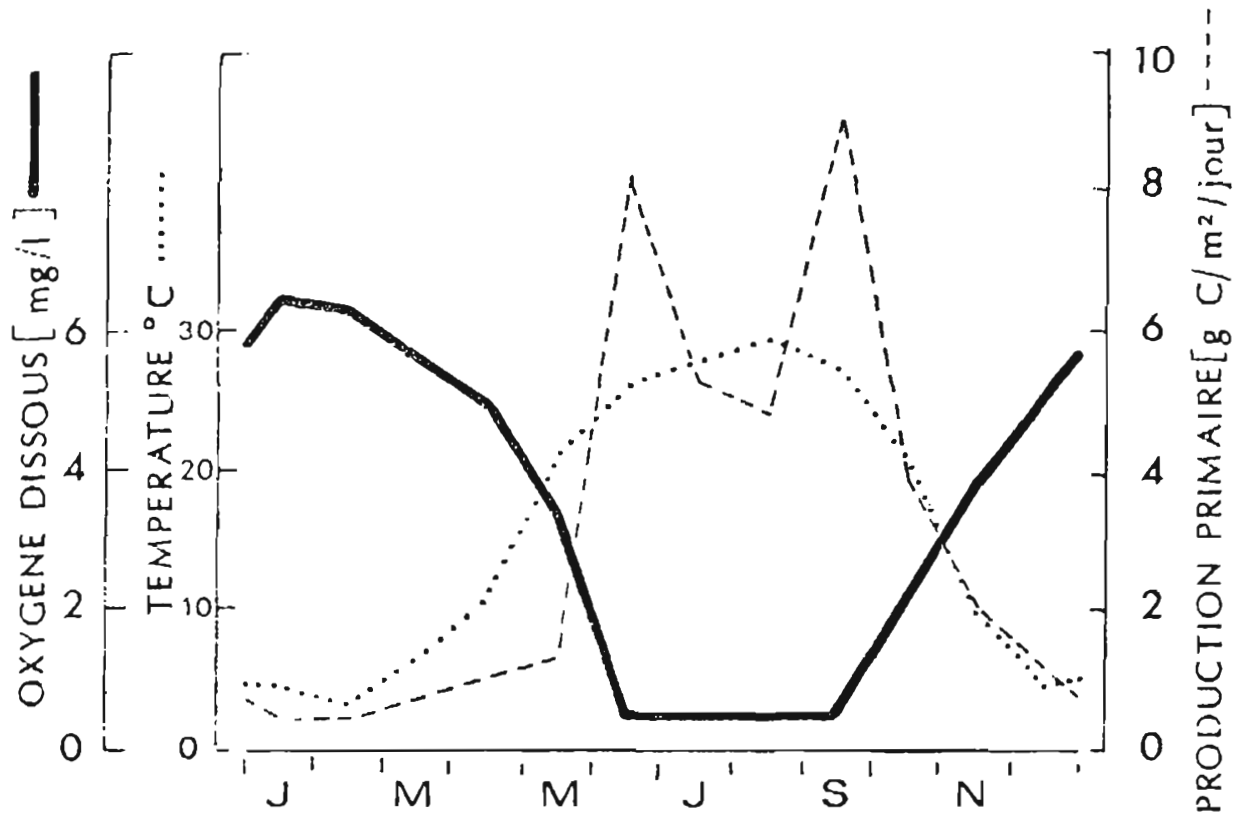

Fig. 11. - Cycles saisonniers moyens de la température, la production primaire et l'oxygene dissous dans la portion médiane de la Chesapeake Bay.

Fig. 11. - Mean seasonal cycles for temperature, primary production and dissolved oxygen in the midportion of Chesapeake Bay. 
difficile, voire impossible. Les modèles numériques peuvent par contre servir à comprendre les épisodes récurrents de baisse d'oxygène dissous (hypoxies) qui, sans atteindre le seuil létal, n'en fragilisent pourtant pas moins la plupart des espèces benthiques (Rosenberg, 1985). C'est ce qui sera illustré par la modélisation de l'eutrophisation de la Baie de Vilaine (Chapelle, 1991).

\subsection{Le modèle écologique}

Comme pour la prolifération des macroalgues, mais cette fois sur une étendue plus vaste et des profondeurs atteignant la dizaine de mètres, le confinement des masses d'eau par l'absence de circulation résiduelle est un préalable nécessaire à l'eutrophisation à phytoplancton. Un modèle hydrodynamique tridimensionnel (Salomon et Lazure, 1988) a confirmé ce fait pour la baie de Vilaine (fig. 12) et permis de calculer les flux advectifs à travers un découpage de la baie en 7 compartiments, dont 3 couples surface/fond. Le modèle écologique utilisé (Chapelle, 1991) nécessite cette fois onze variables d'état pour simuler les cycles de $N$ et $P$, ainsi que l'oxygène dissous (fig. 13).

\subsection{Les résultats}

Après avoir été calibré sur la base de données acquises pour l'année 1984 (fig. 14), le modèle peut être utilisé d'abord pour hiérarchiser les différents processus "consommateurs" d'oxygène dissous: on montre ainsi que c'est la consommation d'oxygène dans la tranche d'eau (respiration et dégradation aérobie du phytoplancton) qui est la principale cause des hypoxies, bien avant la consommation par les processus benthiques, et même avant la diminution de la réaération par l'établissement d'une pycnocline. Le modèle peut aussi être utilisé pour étudier l'effet de réductions des apports azotés et/ou phosphorés sur la production de phytoplancton : les figures $15 a$ et $15 b$ (Chapelle, 1991) montrent clairement qu'au printemps, une réduction du phosphore ou de l'azote dans les mêmes proportions conduirait aux mêmes effets alors qu'en été seul l'azote est limitant. II en résulte que les anoxies, se produisant plutôt en été du fait de la stratification plus stable, seraient donc rendues moins probables par une réduction des apports estivaux d'azote.

\section{DISCUSSION : QUELS MODĖLES POUR L'EUTROPHISATION COTIĖRE ?}

L'eutrophisation des lacs a entraîné dans les années 70 une importante réflexion sur les outils scientifiques d'aide à la décision en matière de stratégie de restauration de la qualité de l'eau. Grossièrement, on peut distinguer deux approches: celle des abaques statistiques introduites par Vollenweider (1968) et celle des modèles écologiques numériques. Par- 
tant du constat que l'élément nutritif limitant de la production végétale en eau douce était le phosphore, et que l'efficacité d'utilisation des apports inorganiques par la biomasse végétale était conditionnée par des facteurs physiques (temps de résidence et éclairement de la tranche d'eau), Vollenweider a classé les lacs dans un repère à deux dimensions : rapport profondeur moyenne/temps de résidence en abscisse et flux annuel de phosphore en ordonnée. La détermination empirique d'une droite sépa-

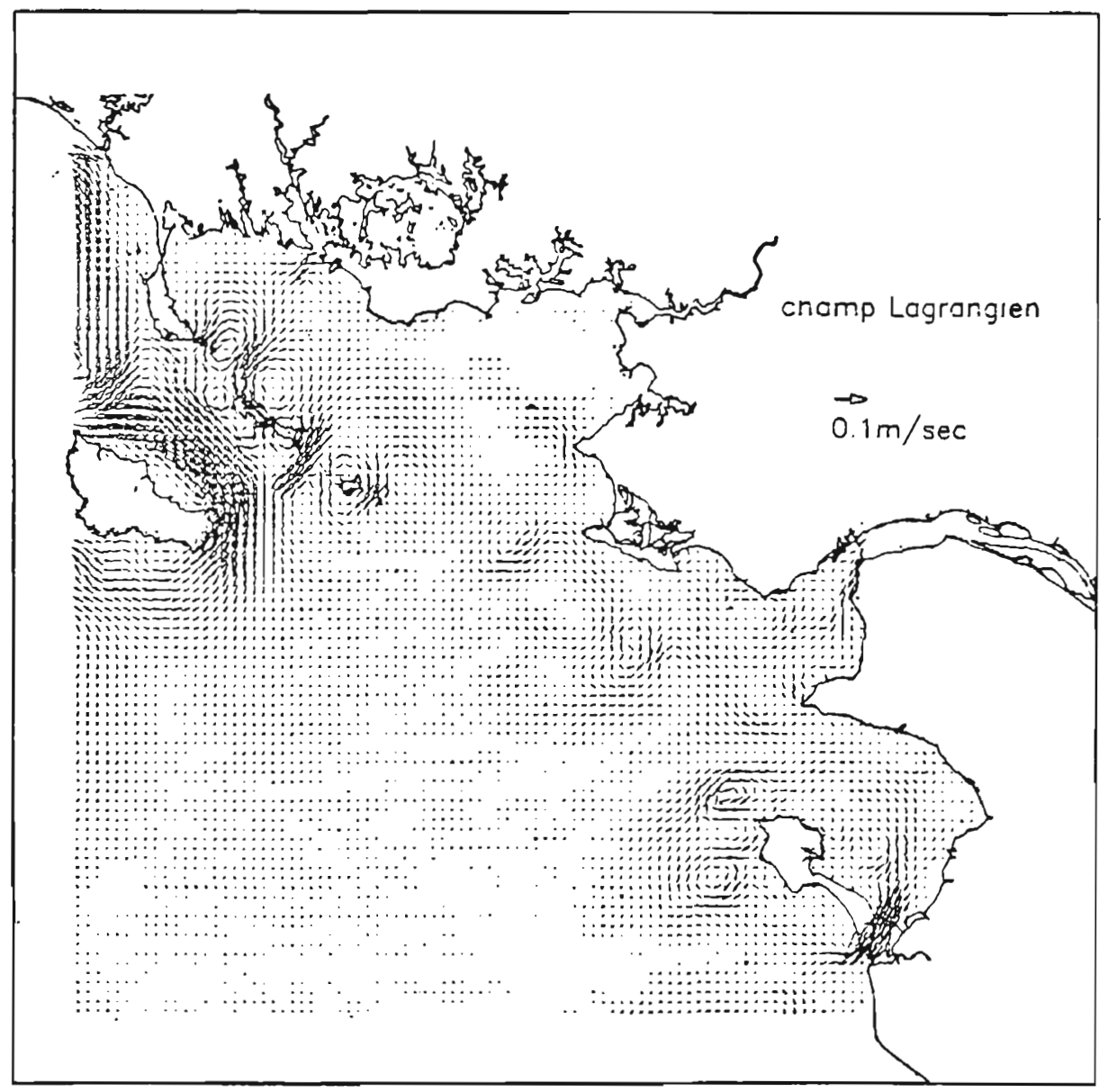

Fig. 12. - Circulation résiduelle de marée en Baie de Vilaine.

Fig. 12. - Tidal residual circulation in the Bay of Vilaine. 


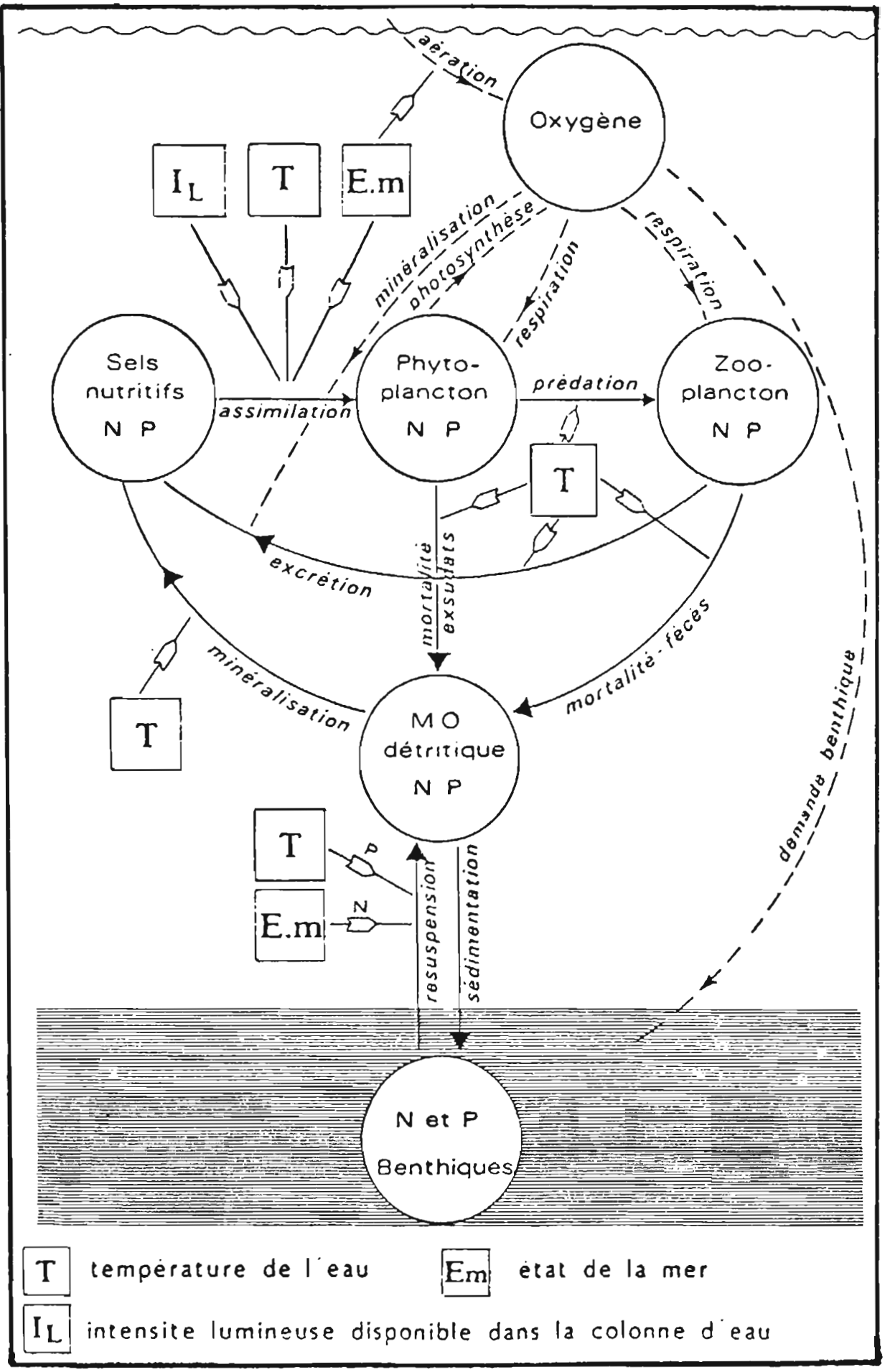

Fig. 13. - Schéma fonctionnel du modèle écologique de la Baie de Vilaine.

Fig. 13. - Flow diagram of the ecological model of the Bay of Vilaine. 


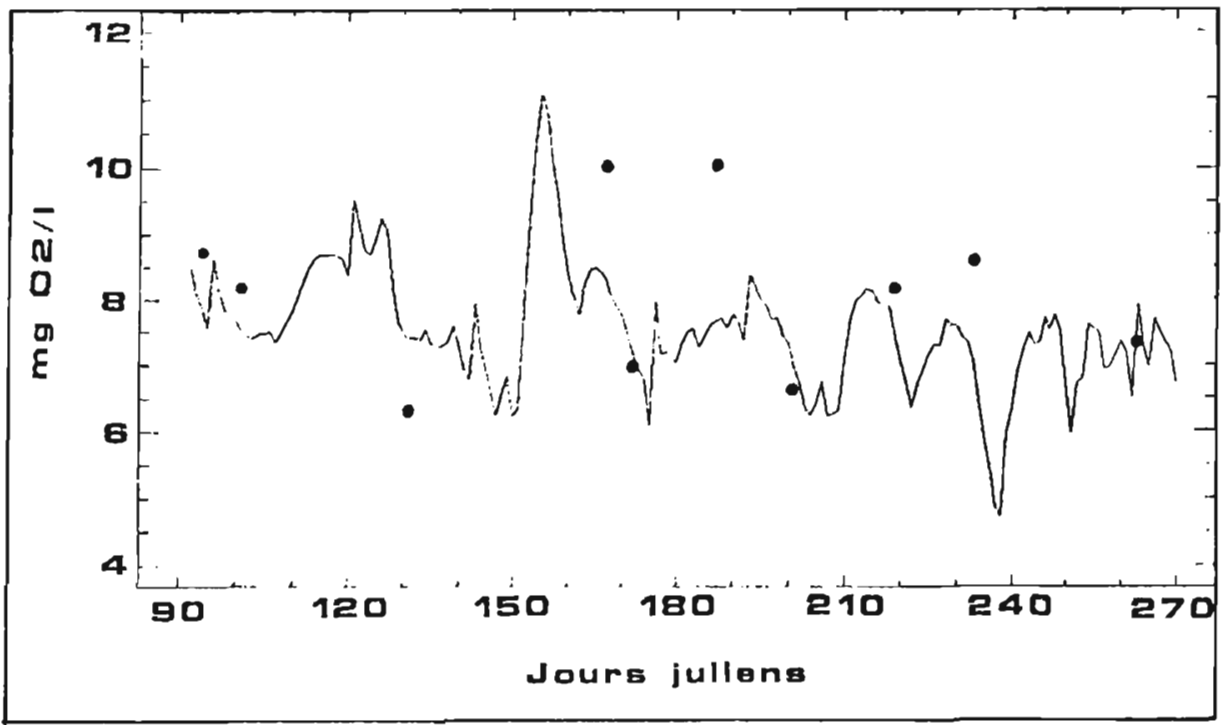

Fig. 14. - Evolution saisonnière mesurée et simulée de la concentration journalière moyenne d'oxygène dissous en 1984 dans un compartiment de fond au milieu de la Baie de Vilaine.

Fig. 14. - Observed and simulated 1984 seasonal evolution of the daily mean of dissolved oxygen in a bottom box in the central Bay of Vilaine.

rant sur ce graphique les lacs dits eutrophes des autres fait de cet abaque un outil opérationnel très simple pour connaître l'état de tout lac. L'OCDE a donc développé cette approche, construisant même des abaques dérivés (Rast et Lee, 1978) permettant de déterminer par exemple la concentration d'oxygène dissous dans des eaux de fond en fonction des critères précédents (fig. 16). Lee et Jones (1981) proposent d'étendre l'usage de ces abaques aux estuaires tout en reconnaissant la plus grande difficulté d'établir un temps de résidence dans une zone aquatique largement ou- verte, ainsi que la possibilité de passage à une limitation nutritive par l'azote dans certains estuaires. Le problème se trouve amplifié en milieu littoral ouvert, ou il n'existe pas de volumes d'eau matériellement délimités, donc pas de temps de résidence facilement déterminables. Le rôle des modèles hydrodynamiques de circulation apparaît donc crucial pour les études d'eutrophisation côtière, de même que la détermination in situ du nutrient limitant. Si l'azote semble être l'élément limitant de la prolifération des macroalgues vertes, la situation est plus nuancée pour le phytoplanc- 


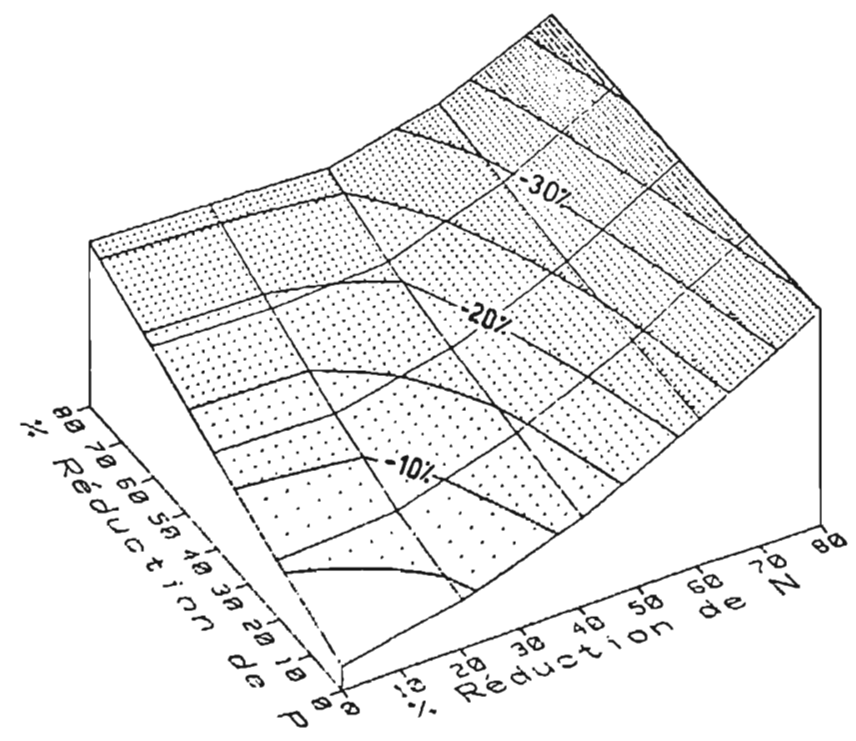

(a)

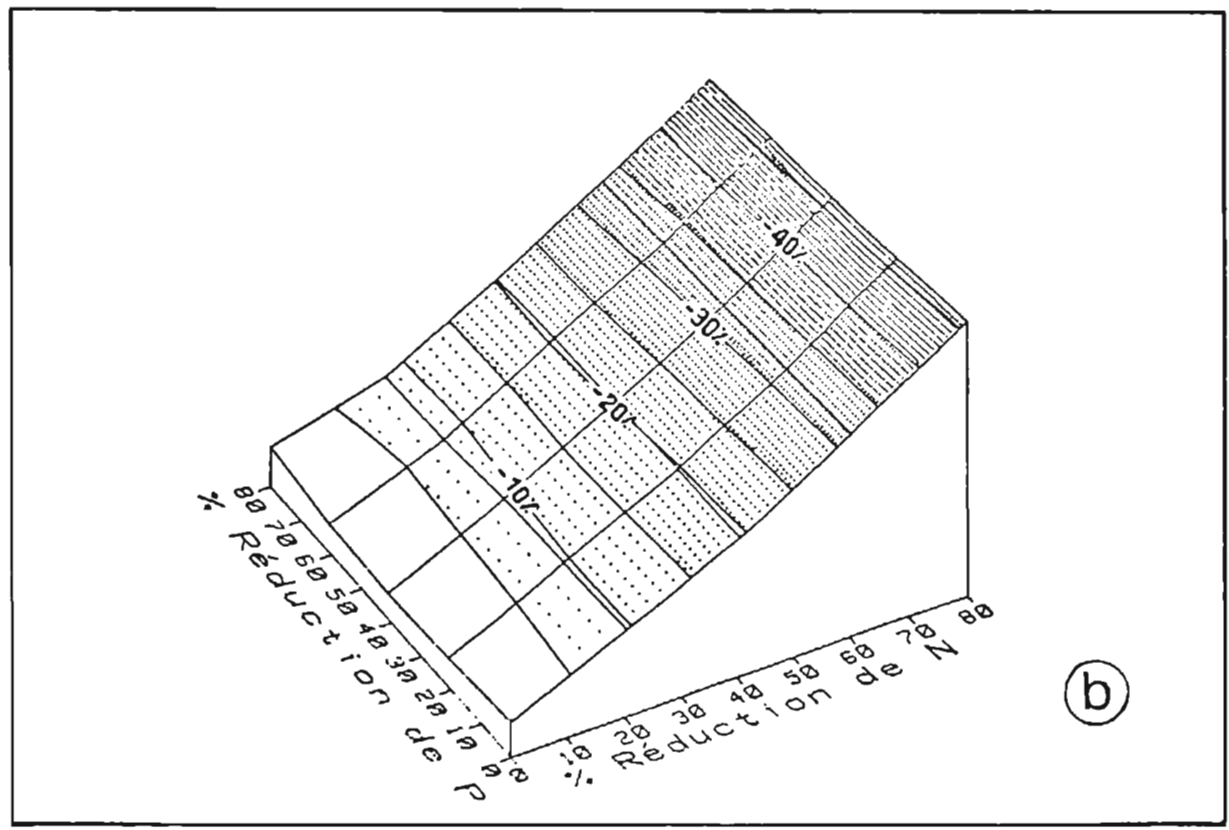

Fig. 15. - Réduction de la biomasse phytoplanctonique moyenne (\%) causée par différents taux de réduction des apports de $\mathrm{N}$ etjou $\mathrm{P}$ par la Vilaine a/ au printemps b/ en été.

Fig. 15. - Reduction of the mean spring phytoplanktonic biomass (\%) caused by various reduction rates of $\mathrm{N}$ and/or $\mathrm{P}$ loadings from the river Vilaine a/during spring b/during summer. 


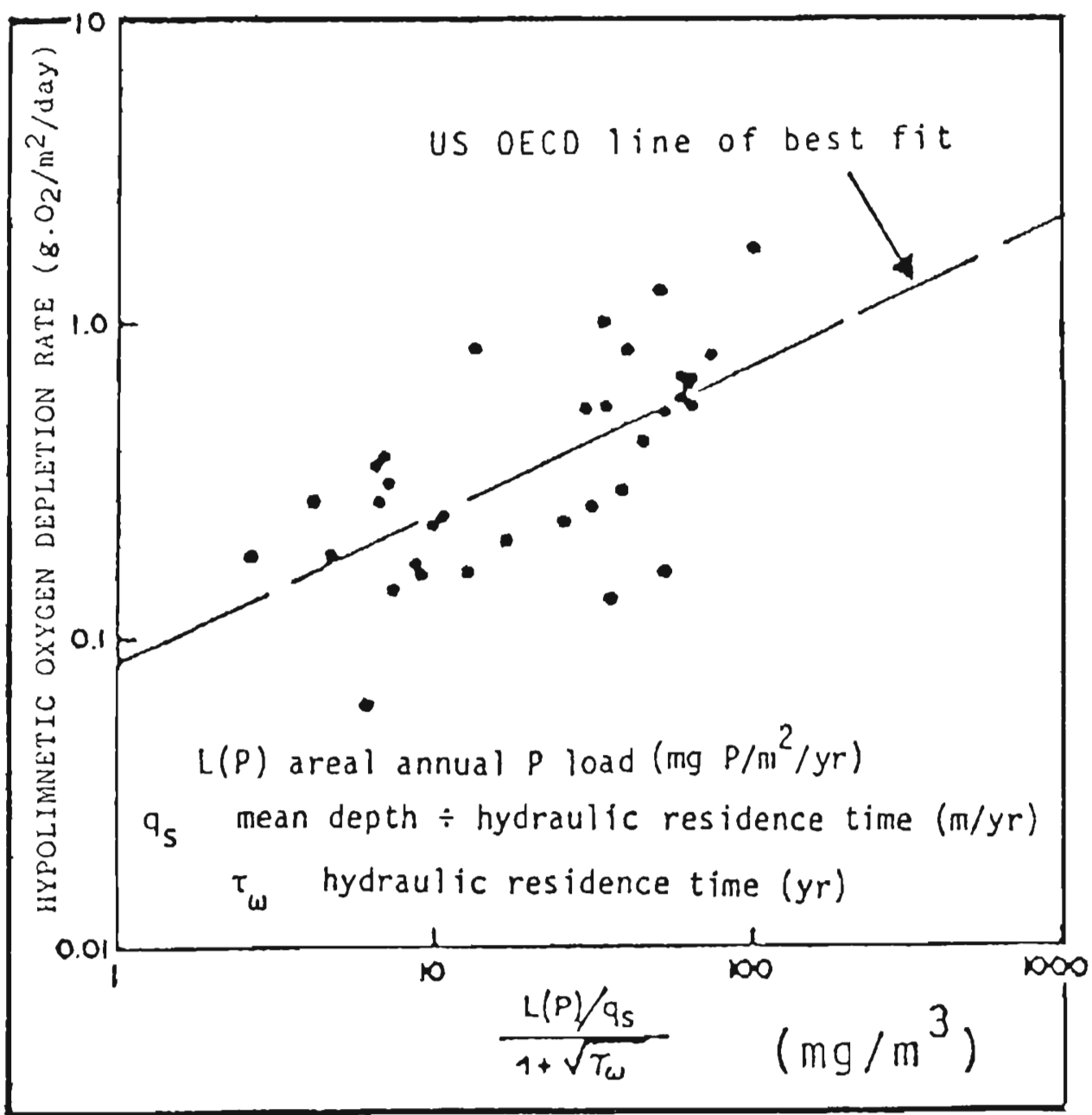

Fig. 16. - Relation empirique entre le taux moyen de désoxygénation de l'hypolimnion des lacs en fonction des caractéristiques hydrauliques et des apports en phosphore.

Fig. 16. - Empirical relationship between mean hypolimnetic oxygen depletion rate of lakes and hydraulic, characteristics and $P$ load.

ton: Ryther et Dunstan (1971) ont clairement montré la limitation par l'azote au large de New-York, alors que Queguiner (1988) a montré l'évolution d'une limitation printanière par le phosphore vers une limitation tardi-estivale par l'azote en Baie de Vilaine, ce qu'a confirmé le modèle écologi- 
que décrit précédemment (Chapelle, 1991). Smayda (1990) évoque par ailleurs le rôle croissant de la limitation des diatomées par le silicium en Mer du Nord au fur et à mesure que l'enrichissement en $\mathrm{N}$ et $\mathrm{P}$ progresse, et le rend responsable de la prédominance croissante des dinoflagellées (non-siliceux) sur les diatomées. La souplesse des modèles biologiques numériques semble bien adaptée à l'étude de ces divers cas d'eutrophisation marine, permettant de dégager progressivement la structure minimale nécessaire en termes de variables d'état pour représenter correctement le phénomène. D'ores et déjà, ces modèles peuvent fournir de très utiles appréciations sur l'effet quantitatif de diverses stratégies de réduction d'apports terrigènes; il semble qu'il faille attendre encore de substantielles améliorations des connaissances pour qu'ils puissent par contre prévoir aussi les changements qualitatifs, tels les remplacements d'espèces par exemple.

\section{BIBLIOGRAPHIE}

Baden S.P., Loo O., Pihl L., \& Rosenberg R., 1990. Effects of eutrophication on benthic communities including fish: Sweedish West Coast. Ambio., 19 (3): 113-122.

Chapelle A., 1991. Modélisation d'un écosystème marin côtier soumis à l'eutrophisation: la baie de Vilaine (Sud-Bretagne). Etude du phytoplancton et du bilan en oxygène. Thèse Univ. Paris VI, $201 \mathrm{p}$.

Dethleisen V. \& Von Westerhagen H., 1983. Oxygen deficiency and effects on bottom fauna in the eastern German Bight, 1982, ICES, CM, 1983, L: 2, $23 \mathrm{p}$.

Dion P., 1988. Étude de certains aspects écophysiologiques des marées vertes. Rapport de contrat CEVA/Pleubian, 37 $\mathrm{p}$.

Di Toro D.M. \& Connolly J.P., 1980. Mathematical models of water quality in large lakes. Part. II: Lake Erié. EPA600/3-80-065. Env. Res. Lab. Duluth MN 55804, $231 \mathrm{p}$.

Droop M.R., 1970. Vitamin $B_{12}$ and marine ecology. V. Continuous culture as an approach to nutritional kinetics Helgol. wiss. Meeresunters. 20: 629-636.

Fransz H.G. \& Verhagen J.H.G., 1975. Modelling research on the production cycle of phytoplankton in the Southern Bight of the North Sea in relation to riverborne nutrient loads. Neth. J. Sea Res., 19: 241-250.

Fujita R.M., 1985. The role of nitrogen status in regulating transient ammonium uptake and nitrogen storage by macroalgae. J. Exp. Mar. Biol. Ecol. 92: 283-301.

Gosse P., Lascombe C. \& Salleron J.L., 1990. Modélisation de la qualité des grands cours d'eau. Eutrophisation: cas de la Moselle et du Doubs. La Houille Blanche, $n^{\circ}$ 3/4: 187-194.

Humphries R.B., Homberger G.M., Spear R.C. \& Mc Comb A.J., 1984. Eutrophication in Peel Inlet - III. A model for the nitrogen scenario and a retrospective look at the preliminary analysis. Water Res. 18 (4) : 389-395.

Jorgensen S.E., 1980. Lake management (Water development, supply and management; vol. 14); Pergamon Press, Oxford, $167 \mathrm{p}$

Kelly M. \& Naguib M., 1984. Eutrophication in coastal marine areas and lagoons: a case study of "Lac de Tunis". Unesco reports in marine science $\mathrm{Nr} 29,54 \mathrm{p}$.

Lee G.F. \& Jones R.A., 1981. Application of the OECD eutrophication modeling 
approach to estuaries. In: Estuaries and nutrients, Neilson S.J. \& Cronin L.E., eds. Humana Press, Clifton (New-Jersey): 549-568.

Lehay D., 1989. Étude de l'hydrologie et I'hydrodynamique de la baie de SaintBrieuc. Approche du rôle de l'hydrodynamisme sur la coquille Saint-Jacques. Thèse de doctorat. Univ. Bretagne Occidentale, Brest, 338 p.

Letts E.A. \& Richards E.H., 1911. Report on green seaweeds and especially UIva latissima in relation to the pollution of the waters in which they occur. Royal Commission on Sewage Disposal. Th Report, HMSO, London. Appendix III, Section II.

Malmgren-Hansen A., Mortensen P. \& Moller B., 1985. Modelling of oxygen depletion in coastal waters. Wat. Sci. Tech. 17: 967-978.

Ménesguen A., 1992. Modelling coastal eutrophication: the case of French Ulva mass blooms. The Science of the Total Environment, Suppl. 1992, 979992.

Ménesguen A. \& Salomon J.C., 1988. Eutrophication modelling as a tool for fighting against Ulva coastal mass blooms. In: Computer modelling in ocean engineering. Schrefler B.A. \& Zienkiewicz O.C., eds. Balkema, Rotterdam: 443-450.

Merceron M., 1987. Mortalités de poissons en Baie de Vilaine (Juillet 1982). Causes, mécanismes, propositions d'actions. Rapport IFREMER/DERO87. 14-99 p.

Mommaerts J.P., Pichot G., Ozer J., Adam Y. \& Baeyens W., 1984. Nitrogen cycling and budget in Belgian coastal waters: North Sea areas with and without river inputs. Rapp. P.V. Réun. Cons. Int. Explor. Mer, 183: 57-69.

Officer C.B., Biggs R.B., Taft J.L., Cronin L.E., Tyler M.A. \& Boynton W.R., 1984. Chesapeake Bay anoxia: origin, development and significance. Science, 233: $22-27$.
Oudin L.C., 1990. Modélisation de l'eutrophisation en Loire. La Houille Blanche, $n^{\circ}$ 3/4 : 195-206.

Park R.A., 1978. A model for simulating lake ecosystems Rept. 3. Center for Ecological Modelling-Rensselaer PoIytechnic Institute. Troy. New-York 12181, $19 \mathrm{p}$.

Piriou J.Y. \& Ménesguen A., 1990. Environmental factors controlling the Ulva sp. blooms in Brittany (France). In: Marine Eutrophication and Population Dynamics, G. Colombo et al., eds., Proc. 25 ${ }^{\text {th }}$ E.M.B.S., Olsen \& Olsen (Denmark) publ.: 111-115.

Piriou J.Y., Ménesguen A. \& Salomon J.C., 1989. Les marées vertes à ulves: conditions nécessaires, évolution et comparaison de sites. In: "Estuaries and coasts: spatial and temporal intercomparisons ", $19^{\mathrm{eme}}$ symposium de l'Estuar. Coast. Sci. Assoc. Elliott M. \& Ducrotoy J.P., eds. 4-8 sept. 1989, Caen (France): 117-122.

Queguiner B., 1988. Synthèse opérationnelle du programme pluriannuel 19831988 de la Commission Quadripartite chargée de l'Aménagement Halieutique et de la Protection Hydrobiologique de la Baie de Quiberon-Vilaine. Rapport Univ. Bretagne Occidentale, Brest $34 \mathrm{p}$.

Rast W. \& Lee G.F., 1978. Summary analysis of the North American (US portion) OECD Eutrophication Project: Nutrient loadings-lake response relationships and trophic state indices. EPA-600/3-78-008.

Rosenberg R., 1985. Eutrophication. The future marine coastal nuisance? Mar. Poll. Bull. 16 (6) : 227-231.

Ryther J.H. \& Dunstan W.M., 1971. Nitrogen, phosphorus and eutrophication in the coastal marine environment, Science 171: 1008-1013.

Salomon J.C., Guegueniat P., Orbi A. \& Baron Y., 1988. A lagrangian model for long term tidally induced transport and mixing. Verification by artificial radionucleide concentrations. In: Radionu- 
cleides: a tool for oceanography, Guary J.C., Guegueniat P. \& Pentreath R.I., eds., Elsevier Applied Science: 384-394.

Salomon J.C. \& Lazure P., 1988. Étude par modèle mathématique de quelques aspects de la circulation marine entre Quiberon et Noirmoutier. Rapport IFREMER/DERO-88.26-EL, 104 p.

Sawyer C., 1965. The sea-lettluce problem in Boston Harbor. J. Wat. Poll. Control Federation, 37: 1122-1133.

Schindler D.W., Fee E.J. \& Ruszczynski, 1978. Phosphorus input and its consequences for phytoplankton standing crop and production in the Experimental Lakes Area and in similar lakes. J. Fish. Res. Bd. Can. 35: 190-196.

Síriso A., Pavoni B. \& Marcomini A., 1989. Macroalgae and phytoplankton standing crops in the central Venice Lagoon; primary production and nutrient balance. The Science of the Total Environment, 80: 139-159.

Smayda T.J., 1990. Novel and nuisance phytoplankton blooms in the sea: evidence for a global epidemic. In: Toxic marine phytoplankton, Graneli E., An- derson D.M., Edler L. \& Sundstrom B. eds. Elsevier Science Publishing: 2940.

Stachowitsch M., 1984. Mass mortality in the Gulf of Trieste: the course of community destruction. P.S.Z.N.I. Marine Ecology, 5 (3): 243-264.

Steffensen D.A., 1976. The effect of nutrient enrichment and temperature on the growth in culture of Ulva lactuca L. Aquat. Bot., 2: 337-351.

Swanson R.L. \& Sindermann C.J., 1979. Oxygen depletion and associated benthic mortalities in New-York Bight, 1976. NOAA Professional Paper, 11, $345 p$.

Thomann R.V., Di Toro D.M., Winfield R.P. \& O'Connor D.J., 1975. Mathematical modelling of phytoplankton in Lake Ontario. 1. Model development and verification. EPA-660/375-005, ORD, Corvallis, Oregon, $177 \mathrm{p}$.

Vollenweider R.A., 1968. the scientific basis of lake and stream eutrophication, with particular reference to phosphorus and nitrogen as factors in eutrophication. Tech. Rept. to OCDE, Paris. DAS/CSI/68-27, 182 p. 